\title{
Characterization of Peripheral Neuropathy in Rat Model of Type 2 Diabetes
}

\author{
Bina Kumari Mehta, Damini Nerkar, 'Sugato Banerjee \\ Department of Pharmaceutical Sciences and Technology, Birla Institute of Technology, Mesra, Ranchi, INDIA.
}

\begin{abstract}
Diabetic neuropathy, a microvascular complication associated with diabetes, is one of the most common forms of neuropathy. Current rodent models of type 2 diabetes are mostly transgenic which fail to mimic human type 2 diabetes and its secondary complications, including peripheral neuropathy. Our aim is to develop a non-transgenic animal model of type 2 diabetic neuropathy which closely mimics the human disease. Methods: High-fat diet (HFD; normal pellet diet + lard) and a dual dose of streptozotocin $(25 \mathrm{mg} / \mathrm{kg}$ ) were used to induce diabetes in Sprague-Dawley rats. Developments of neuropathy in these animals were measured using behavioral parameters like tail flick latency, pain threshold using randall selitto, and gait test. Nerve conduction velocity and histopathological evaluation of sciatic nerve were also carried out. Paclitaxel treated animals served as neuropathy controls. Results: The animals developed diabetes (blood glucose $>250 \mathrm{mg}$ / $\mathrm{dl} ; \mathrm{HbA} 1 \mathrm{c} 11.77 \pm 0.14 \%)$. All the classical symptoms of painful neuropathy, reduction in tail flick latency $(p<0.05)$, reduced vocalization threshold in randall selitto $(p<0.01)$, gait test showing a highly negative sciatic functional index $(p<0.01)$ and reduced nerve conduction velocity $(p<0.01)$ were evident in HFD-STZ animals. The above response was comparable to paclitaxel-treated neuropathy controls. Histopathological evaluation of sciatic nerves showed indications of sciatic nerve damage in HFD-STZ and paclitaxeltreated animals. The above neuropathic conditions were successfully reversed upon glibenclamide treatment $(10 \mathrm{mg} / \mathrm{kg})$ in HFD-STZ treated diabetic animals. Conclusion: Hence we successfully developed a cost-efficient non-transgenic model of diabetic neuropathy which closely mimics the characteristics of the human form of the disease.
\end{abstract}

Key words: Animal model, Diabetes, Neuropathy, Glibenclamide, Rat.

\section{INTRODUCTION}

Diabetes mellitus is a group of metabolic disorders characterized by hyperglycaemia resulting from defects in insulin secretion, insulin action or both. Diabetes is associated with long-term damage, dysfunction and eventually failure of various organs especially eyes, kidneys, nerves, heart and blood vessels. Diabetic neuropathy, a microvascular complication associated with diabetes, is one of the most common forms of neuropathy. It manifests in $28 \%$ to $55 \%$ of patients with pain. ${ }^{1,2}$ Patients with type 1 or type 2 diabetes are at a greater risk of developing diabetic neuropathy. Peripheral neuropathy is the most prevalent form of neuropathy, represented by a complex network of interrelated metabolic, neurotropic and vascular defects. It leads to chronic progressive damage and loss of unmyelinated and myelinated nerve fibers which usually culminate into distal symmetric axonal sensorimotor neuropathy. ${ }^{3}$ Slow nerve conduction velocity (NCV), thermal hypoalgesia, tactile allodynia, upregulation of markers of oxidative stress and increased sciatic nerve glucose, sorbitol and fructose are standard markers of neuropathy in murine models of type 2 diabetes. Animal models must replicate the phenotype as well as the disease pathogenesis seen in patients as closely as possible. From a practical perspective, models that are easy
Submission Date: 16-09-2016; Revision Date: 17-11-2016; Accepted Date: 23-11-2016

DOI: 10.5530/ijper.51.1.13 Correspondence: Sugato Banerjee, Department of Pharmaceutical Sciences and Technology, Birla Institute of Technology, Mesra, Ranchi: 835215, India

Phone: 91-651-2275444

Fax: 91-651-2275290

E-mail: sbanerjee@bitmesra. ac.in

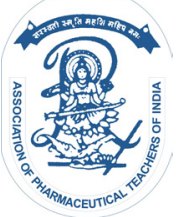

www.ijper.org 
to maintain, economical and develop faster are always favored. No animal model is perfect, and current rodent models of type 2 diabetes have been shown to have some limitations (reviewed by Bugger and Abel). ${ }^{4}$ Most of these models comprise of transgenic animals which are expensive to maintain and also fail to mimic the multifactorial disease pathogenesis process. ${ }^{5} \mathrm{~A}$ rat model first proposed by Reed et al., ${ }^{6}$ and later modified by Srinivasan $e t$ al., ${ }^{7}$ aimed to induce type 2 diabetes using high-fat diet (HFD) resulting in peripheral insulin resistance, followed by a low dose of the pancreatic $\beta$-cell toxin, streptozotocin (STZ). STZ is commonly used at high doses to induce type 1 diabetes, as it destroys $\beta$-cell resulting in impaired insulin secretion. ${ }^{8,9}$ A low dose of STZ after HFD; modestly impairs the function of the $\beta$-cells, resulting in glucose intolerance. ${ }^{6,7}$ This condition may mimic the human disease process resulting in a metabolic phenotype similar to that in late stage type 2 diabetic patients. Combining HFD and a low dose of STZ treatment successfully induce type 2 diabetes in rats, the dose of STZ and duration of the disease varies between studies. Others reported that STZ might be given in multiple low doses. Zhang and his collogues ${ }^{10}$ first demonstrates that a combination of HFD and multiple low doses of STZ could be used to generate a rat model showing all the characteristics of type 2 diabetes persisting for up to 9 weeks after induction of diabetes. This prolonged hyperglycemia and hyperlipidemia may lead to the development of secondary complications. This study used HFD and a dual dose of STZ $(30 \mathrm{mg} / \mathrm{kg})$ to induce diabetes in wister rats. Davidson et al., ${ }^{11}$ successfully induced diabetic neuropathy in 12 weeks after prolonged HFD (8 weeks) followed by single $30 \mathrm{mg} / \mathrm{kg}$ STZ in SD rats. However, a later study by Mansor et al., ${ }^{12}$ reported that such high dose of STZ might induce type 1 diabetes like features including hypoinsulinemia, hyperkalemia, and weight loss. They suggested $25 \mathrm{mg} / \mathrm{kg}$ be a more appropriate dose that mirrored the pathological and metabolic features of type 2 patients in these animals. Thus all previous animal models of diabetes using $>25 \mathrm{mg} / \mathrm{kg}$ STZ seems to mimic more of the Type 1 subtype.

In the present study, we used HFD (3 weeks) and a dual dose of $25 \mathrm{mg} / \mathrm{kg}$ STZ to induce type 2 diabetes in Sprague-Dawley (SD) rats and study the development of neuropathy in these animals by eight weeks of initiation of the study. We also examined the effect of anti-diabetic compound glibenclamide on diabetic neuropathy in these animals.

\section{MATERIALS AND METHODS}

\section{Animals}

SD rats weighing approximately $160-180 \mathrm{~g}$, initially obtained from National Institute of Nutrition Hyderabad, India were procured from the central animal facility of the Institute (Regd. No. BIT/PH/IAEC/12/2013). The animals were acclimatized for 7 days in the institute's animal house and were housed in standard polypropylene cages. The animals were maintained at controlled room temperature $\left(24-28^{0} \mathrm{C}\right)$ and humidity $(55-60 \% \mathrm{RH})$. All rats were provided with commercially available rat normal pellet diet (NPD; Amrut Diet, New Delhi, India) and water ad libitum, prior to the dietary manipulation. The guidelines of committee for the purpose of control and supervision of experiments on animals (CPCSEA), Govt. of India were followed and prior permission was sought from the institutional animal ethics committee for conducting the study.

\section{Development of type 2 diabetic model}

The animals were divided into three groups $(n=10 /$ group)

\section{Control group}

The control group was fed with Normal Pellet Diet (NPD) and water ad libitum. The control group was administered with vehicle (citrate buffer, $\mathrm{pH}-4.5$ ) in a dose volume of $1 \mathrm{ml} / \mathrm{kg}$, IP.

\section{Neuropathy controls group}

Paclitaxel group was fed with normal pellet diet (NPD) and given water ad libitum. The paclitaxel group received intra-peritoneal injections of paclitaxel $(1 \mathrm{mg} / \mathrm{kg})$ on four alternate days viz 0, 2, 4, 6 days. Evaluations were done on every $7^{\text {th }}, 14^{\text {th }}, 21^{\text {st }}$ and $28^{\text {th }}$ day..$^{13,14}$

\section{Diabetic group}

HFD-STZ group was fed with High Fat Diet (NPD + Lard 310 gm/kg; Baroda Earth Private Limited, Baroda India) for 3 weeks followed by a low dose of STZ $(25 \mathrm{mg} / \mathrm{kg})$. After one week, FBG of HFD-STZ rats were measured and the rats with FBG $>200 \mathrm{mg} / \mathrm{dl}$ (success rate $90 \%$ ) were again administered with a low dose of STZ $(25 \mathrm{mg} / \mathrm{kg})$. Evaluations for symptoms of neuropathy for test group (HFD-STZ) started 2 weeks after induction of diabetes and were carried up to 12 weeks. The high-fat diet was continued for the entire period of study.

\section{Treatment group}

After induction of diabetes, animals were allowed to maintain increased blood glucose levels for 3 weeks (week 4 to week 7). This was followed by 3 weeks of 
treatment with glibenclamide $(10 \mathrm{mg} / \mathrm{kg})$ for both the above animals as well as paclitaxel-treated animals.

\section{Fasting blood glucose levels}

Blood was withdrawn from the tail vein of the animals, and the blood glucose level was checked using a calibrated glucometer (Model No B 1801 Contour TS; Bayer Diabetes Care India) every week from the initiation of study till animals were sacrificed. HbA1c (Gluquant A1c; Meril Life Sciences Pvt. Ltd) levels were also measured for HFD-STZ and control animals 2 weeks after induction of diabetes and after glibenclamide treatment. HbA1c was also measured for STZ $(25 \mathrm{mg} / \mathrm{kg}$ dual dose), paclitaxel and paclitaxel glibenclamide treated animals.

\section{Oral glucose tolerance test}

Animals were fasted for $12 \mathrm{~h}$ prior to OGT'T (oral glucose tolerance test). At 0 hours before glucose administration, blood glucose was measured by tail prick method using one touch glucose strips (One Touch Ultra, Life Scan Inc). Glucose solution $2 \mathrm{~g} / \mathrm{kg}$ ( $40 \%$ aqueous solution) was administered by oral gavage. After glucose administration, circulating glucose levels were measured at 30 , 60, 90 and $120 \mathrm{~min}$ by the above method and area under the curve (AUC) was calculated.

\section{Lipid profile}

The blood for carrying out lipid profile tests was collected by the retro-orbital method. The serum was separated from the plasma by centrifugation (Remi Industries Ltd, Mumbai, India) at $15000 \mathrm{rpm}$ for 10 minutes. The serum thus obtained was then used for further analysis. Triglyceride, total cholesterol, HDL, and LDL cholesterol was measured using kit as per manufacturer's instructions (Span Diagnostics; Span Diagnostics Ltd. Surat, India)

\section{Blood pressure}

Blood pressure (BP) of animals was recorded 4 weeks after induction of diabetes using a tail cuff blood pressure system (Non - invasive blood pressure by IITC model No. 229) equipped with pulse amplifier with tail cuff sensor. This method requires minimal warming of rats to $30^{\circ} \mathrm{C}$ in warming chamber. The built-in cuff pump regulates cuff pressure limits. Rats were acclimatized in the blood pressure equipment restrainer for 4 days before measurement and then blood pressure was measured.

\section{Behavioral evaluation}

Tail Flick Method - The tail was immersed in water maintained at a temperature of around $55^{\circ} \mathrm{C} \pm 5$. The flicking of the tail was considered as a positive response. To prevent tissue damage, the maximum time for which tail was immersed tail was considered as 15 seconds. For each rat, tail flick latency was measured for three times and the average was reported as tail flick latency. Between every test, rats were left free for half an hour. ${ }^{13}$

\section{Static Mechanical Hyperalgesic Test (Randall-Selitto)}

The nociceptive withdrawal threshold was assessed by using the Randall-Selitto electronic algesimeter (IITC 2500 Digital Paw Pressure Meter, IITC Life Sciences, Woodland Hills, CA). Before the test, each animal received 5 min of handling to get used to manipulation; then it was placed into a soft cotton cloth and carefully immobilized with the same hand used to hold the tested paw. The test consisted of the application of an increasing mechanical force, in which the tip of the device was applied to the medial portion of the plantar or the dorsal surfaces of both fore and hind paws. The point of application was marked with ink in order to maintain the location of repeated trials. The maximum force applied was limited to $250 \mathrm{~g}$ to avoid skin damage. ${ }^{13,15}$

\section{Gait Test}

Walking Track analysis or Gait Test assesses nerve function. For evaluating footprints and for calculating the Sciatic Functional Index, the animals were submitted to walk on a $43 \mathrm{~cm}$ long vs. $8.7 \mathrm{~cm}$ wide vs. $5.5 \mathrm{~cm}$ high wooden track, with a small dark shelter at the end. On the walking path, paper strips were applied to the same measurements. Those paper strips containing animal footprints were manually assessed. ${ }^{16}$ Animal paws measurements were highlighted and, with the aid of a school rule, with $1 \mathrm{~cm}$ increments, the values were calculated. ${ }^{17}$ The Sciatic Functional Index (SFI) was calculated according to the following equation: $\mathrm{SFI}=$ -38.3 (EPL-NPL)/NPL + 109.5(ETS-NTS)/NTS + 13.3(EIT-NIT)/NIT - 8.8. PL=distance between the heel and the third toe, TS = distance between the first and fifth toes and IT = distance between the second and fourth toes. $\mathrm{E}$ and $\mathrm{N}$ represented the experimental and normal sides. ${ }^{18}$

\section{Motor nerve conduction velocity}

Six weeks after initiation of hyperglycemia and after glibenclamide treatment MNCV were measured. Animals were anesthetized by injection of Ketamine/Diazepam solution $(50 / 5 \mathrm{mg} / \mathrm{kg}$, IP). The environment temperature was maintained at $25 \pm 1{ }^{\circ} \mathrm{C}$ during all stages of the study. After shaving the animal's back, a small incision was made in left sciatic notch and ankle. Then, by bi-polar electrodes, proximal part of the sciatic notch and the distal part of the ankle were stimulated $(0.5-2 \mathrm{~mA}$ 
for $0.01-3 \mathrm{~ms}$ ) and after stimulation, Motor Nerve Conductivity Velocity (MNCV) of sciatic-tibial was recorded. The obtained records are biphasic responses with a primary $\mathrm{M}$-wave produced due to the stimulation of motor fibers. ${ }^{19,20}$ MNCV was calculated by dividing the distance between the two stimulated sites ( $\mathrm{mm}$ ) by the difference between proximal and distal latencies (ms).

\section{Biochemical Estimation}

At the end of the behavioral studies, the animals were sacrificed by cervical dislocation using ether anesthesia, and the sciatic nerves were removed. They were rinsed thoroughly with $0.9 \% \mathrm{w} / \mathrm{v}$ saline. A $10 \%$ (w/v) homogenate of the tissue was prepared in Tris Buffer $(10 \mathrm{mM}$, $\mathrm{pH}$ 7.4). The homogenate was then used for the determination of reduced glutathione and superoxide dismutase levels.

\section{Reduced Glutathione Estimation}

Reduced Glutathione was estimated by using the method described by Monron et al., ${ }^{21}$ Equal volume of tissue homogenate and 20\% trichloroacetic acid (TCA) were mixed. The precipitated fraction was centrifuged and to $0.25 \mathrm{ml}$ of supernatant, $2 \mathrm{ml}$ of DTNB (5, 5'-dithiobis (2-nitro benzoic acid) reagent was added. The final volume was made up to $3 \mathrm{ml}$ with phosphate buffer. The colour developed was read at $415 \mathrm{~nm}$ against reagent blank. Different concentration of standard glutathione was taken and processed as above for standard graph. The amount of reduced glutathione was expressed as $\mu \mathrm{g}$ of GSH/ mg protein. ${ }^{22}$

\section{Superoxide dismutase (SOD) Estimation}

Superoxide dismutase was estimated by using the method described by Misra and Fridovich. ${ }^{23} 0.5 \mathrm{ml}$ of tissue homogenate was diluted with $0.5 \mathrm{ml}$ of distilled water, to which $0.25 \mathrm{ml}$ of ice-cold ethanol and $0.25 \mathrm{ml}$ of ice cold chloroform was added. The mixture was mixed well using cyclomixer for 5 minutes and centrifuged at $2500 \mathrm{~g}$. To $0.5 \mathrm{ml}$ of supernatant, added $1.5 \mathrm{ml}$ of carbonate buffer and $0.5 \mathrm{ml}$ of EDTA solution. The reaction was initiated by addition of $0.4 \mathrm{ml}$ of epinephrine and the change in $\mathrm{OD} /$ minute was measured at $480 \mathrm{~nm}$ against blank. SOD activity was expressed as units/ $\mathrm{mg}$ of protein. Change in optical density per minute at $50 \%$ inhibition of epinephrine to adrenochrome transition by the enzyme is taken as the enzyme unit. Calibration curve was prepared by using standard Superoxide dismutase. ${ }^{22}$

\section{Histopathological evaluation}

The sciatic nerve was isolated after sacrifice and stored in $10 \%$ formalin. The nerves were then sectioned and stained (Hematoxylin and Eosin). The scanning was done under light microscopy $(10 \times 40 \mathrm{X})$ for axonal degeneration. ${ }^{24}$

\section{Statistical analysis}

The data were evaluated by one-way ANOVA followed by Tukey's multiple comparison tests. $\mathrm{p}<0.05$ was considered to be statistically significant. All values are expressed as Mean \pm SEM. Statistical analysis was carried out using Graph Pad Prism 4.0 software (Graph Pad Software Inc San Diego CA, USA).

\section{RESULTS}

\section{Fasting blood glucose levels and Glucose tolerance}

The animals in control and neuropathy control group of animals showed normal blood glucose levels throughout the experiment. However, the HFD-STZ group animals showed a marked increase in FBG as compared to healthy animals. The FBG levels were $>250 \mathrm{mg} / \mathrm{dl}$ throughout the experiment (10 weeks) for HFD-STZ group. The initial blood glucose levels in HFD animals before induction of $1^{\text {st }} \mathrm{STZ}$ injection in HFD-STZ group was $92.33 \pm 2.4 \mathrm{mg} / \mathrm{dL}$. (Figure 1A). Glucose utilization was also significantly impaired in HFD-STZ-diabetic rats (Figure1B). Mean HbA1c levels for controls were $6.63 \pm 0.09 \%$ while HFD-STZ animals showed higher levels at $11.77 \pm 0.14 \%$. STZ or paclitaxel-treated animals ( $\mathrm{n}=10 /$ group) showed comparable FBG and HbA1c levels to untreated controls (data not shown). Treatment with glibenclamide for 3 weeks reduced FBG to $<150 \mathrm{mg} / \mathrm{dL}$ and HbA1c levels to $6.84 \pm 0.23 \%$ ( $\mathrm{n}=10 /$ group $)$

\section{Lipid profile}

The control and paclitaxel groups had normal triglyceride, total cholesterol, and high-density lipoproteins (HDL) levels. However, there was marked increase in triglyceride and total cholesterol level in animals fed with high fat diet $(p<0.001)$ whereas the HDL level was found to decrease $(p<0.001)$ in these animals, when compared to control and paclitaxel, treated groups of animals (Figure 2). Glibenclamide treatment led to significant reduction in the triglyceride levels $(p<0.05)$ while total cholesterol levels were unaltered as reported previously ( $\mathrm{n}=10 /$ group$^{7}{ }^{7}$

\section{Blood pressure}

However, diabetes, as well as high lipid levels did not affect the BP in these animals. The Systolic and 
A

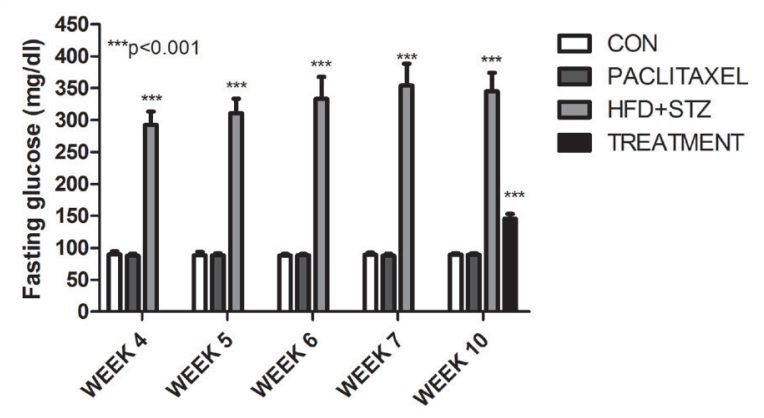

B

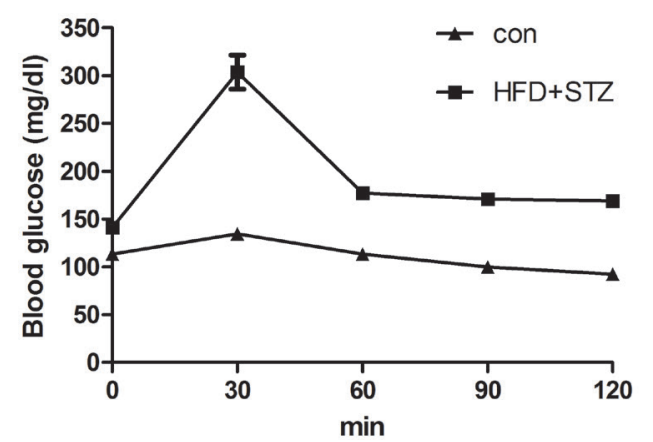

Figure 1: Fasting blood glucose levels and glucose tolerance in type 2 diabetic animals. A) Significant increase in fasting blood glucose level was observed in HFD-STZ after 4 weeks of HFD and a dual dose of STZ $(p<0.001)$ over control and paclitaxel-treated groups. Three weeks of treatment with glibenclamide $(10 \mathrm{mg} / \mathrm{kg})$ normalized the fasting blood glucose levels. B) The area under the curve (AUC) was significantly different $p<0.01$ for HFD-STZ vs.control animals. All values are expressed as mean $\pm S E M n=10$.

Diastolic pressure were found to be 55.67 $\pm 2.3-120 \pm 2.9$ $\mathrm{mm}$ and $56.6 \pm 1.2-116 \pm 3 \mathrm{~mm}$ for control and HFDSTZ respectively, which were comparable.

\section{Tail Immersion Test}

On week 8 after initiation of study non-noxious hot stimuli $\left(55^{\circ} \mathrm{C}\right)$ lead to significantly reduced tail withdrawal latency in paclitaxel $(\mathrm{p}<0.05)$ as previous reported $^{25,26}$ and HFD-STZ groups as compared to control group animals $(p<0.05)$. However, no significant difference was found between Paclitaxel and HFD-STZ Group ( $p>0.05)$. The decrease in the tail withdrawal latency of paclitaxel and HFD-STZ groups indicated hyperalgesia a symptom for an early stage of neuropathy in these animals. ${ }^{27}$ 3-week glibenclamide treatment successfully reversed withdrawal latency (Figure 3A). After 12 weeks of initiation of the study, the animals showed hypoalgesia due to loss of peripheral nerves indicating late stage neuropathy. ${ }^{27}$ Glibenclamide treatment could not reverse this condition (data not shown). Glib- enclamide treatment did not affect paclitaxel-induced hyperalgesia (data not shown).

\section{Static Mechanical Hyperalgesia Test (Randall- Selitto)}

There was a significant decrease in the withdrawal threshold for paclitaxel $(\mathrm{p}<0.05)$ and HFD-STZ $(p<0.01)$ treated animals as compared to control group. Withdrawal time was, however, found to be comparable between paclitaxel and HFD-STZ group. Upon treatment with glibenclamide for three weeks the withdrawal threshold got normalized and became similar to control animals (Figure 3B). However, after 12 weeks of initiation of the study, the animals showed an increase in withdrawal threshold. Glibenclamide treatment could not reverse this (data not shown). Glibenclamide treatment did not affect paclitaxel-induced hyperalgesia (data not shown).

\section{Gait Test}

The paclitaxel and HFD-STZ group animals showed marked changes in the walking pattern with less application of weight on the paws and merging of toe spread. The sciatic functional index) SFI was calculated based on the formula mentioned in the methods section. The SFI between control and HFD-STZ as well as the paclitaxeltreated group was found to be significantly different $(p<0.01 n=10)$. No significant change in SFI was found between paclitaxel and HFD-STZ group (Figure 3C). The toe spread and print length were found to be less well defined while the distance between $2^{\text {nd }}$ and $4^{\text {th }}$ toe was more compressed, an indicator for neuropathy ${ }^{28}$ in HFD-STZ and paclitaxel treated neuropathy groups when compared to control animals. Pictorial representation of the paws of HFD-STZ and Paclitaxel-treated animals showing the physical changes associated with neuropathy (Figure 3D).

\section{Superoxide Dismutase (SOD) and Reduced Glutathione (GSH) levels}

A significant decrease in SOD levels $(\mathrm{p}<0.05 \mathrm{n}=10)$ as well as GSH levels $(\mathrm{p}<0.01 \mathrm{n}=10)$ was observed in Paclitaxel and HFD-STZ as compared to control group. SOD levels were found to be comparable in HFD-STZ and paclitaxel group. (Figure $4 \mathrm{~A}$ and B).

\section{Motor Nerve Conduction Velocity Test (MNCV Test)}

Motor nerve conduction velocity was decreased significantly in paclitaxel $(48 \pm 2.4 \mathrm{p}<0.01 \mathrm{n}=10)$ and HFD-STZ $(45 \pm 3.5 \mathrm{p}<0.01 \mathrm{n}=10)$ as compared to control animals $(58 \pm 3.2 \mathrm{n}=10)$. Glibenclamide treatment successfully reversed the reduced $\mathrm{MNCV}$ in diabetic animals $(55.43 \pm 0.62 \mathrm{n}=10)$ (Figure 4C). 
A

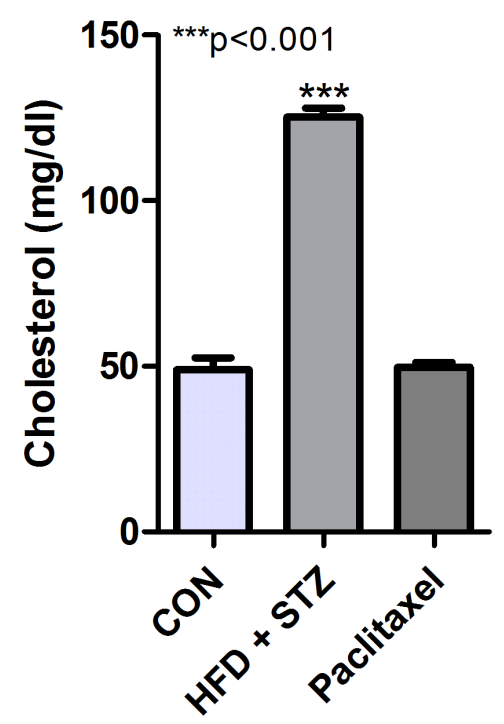

B

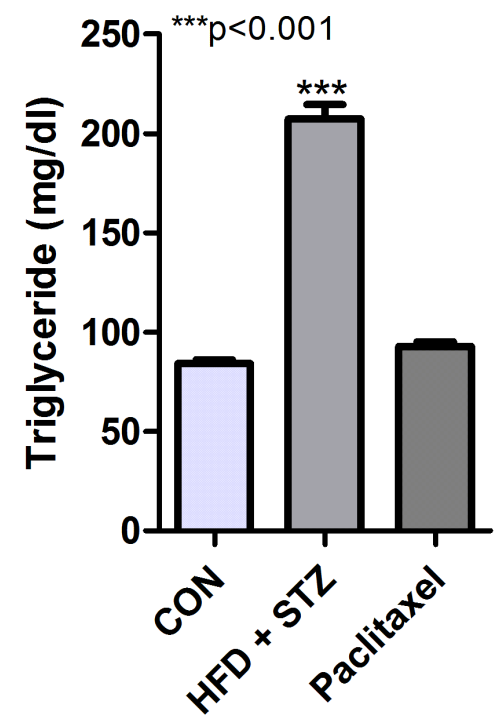

C

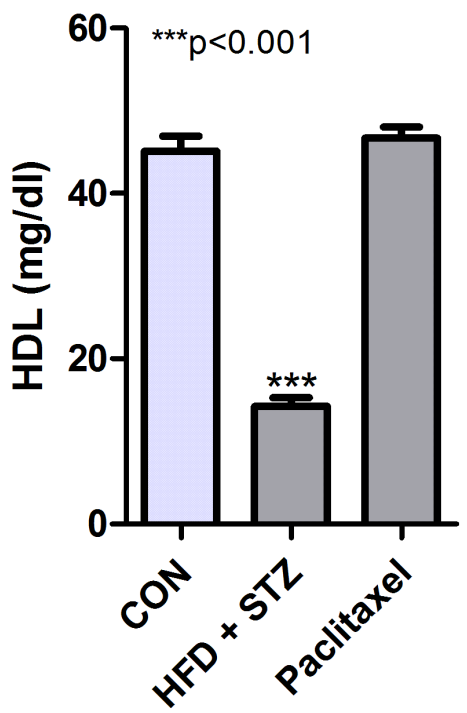

Figure 2: Lipid levels in type 2 diabetic animals. A) Levels of total cholesterol showing significant increase after treatment with HFD-STZ on week 7 when compared to control and paclitaxel; B) Levels of Triglyceride showing significant increase ( $<<0.001$ ) after treatment with HFD-STZ on week 7 compared to control and paclitaxel C) Levels of HDL showing significant decrease $(p<0.001)$ after treatment with HFD+STZ on week 7 control and paclitaxel. All values expressed as mean $\pm S E M$ for $n=10$
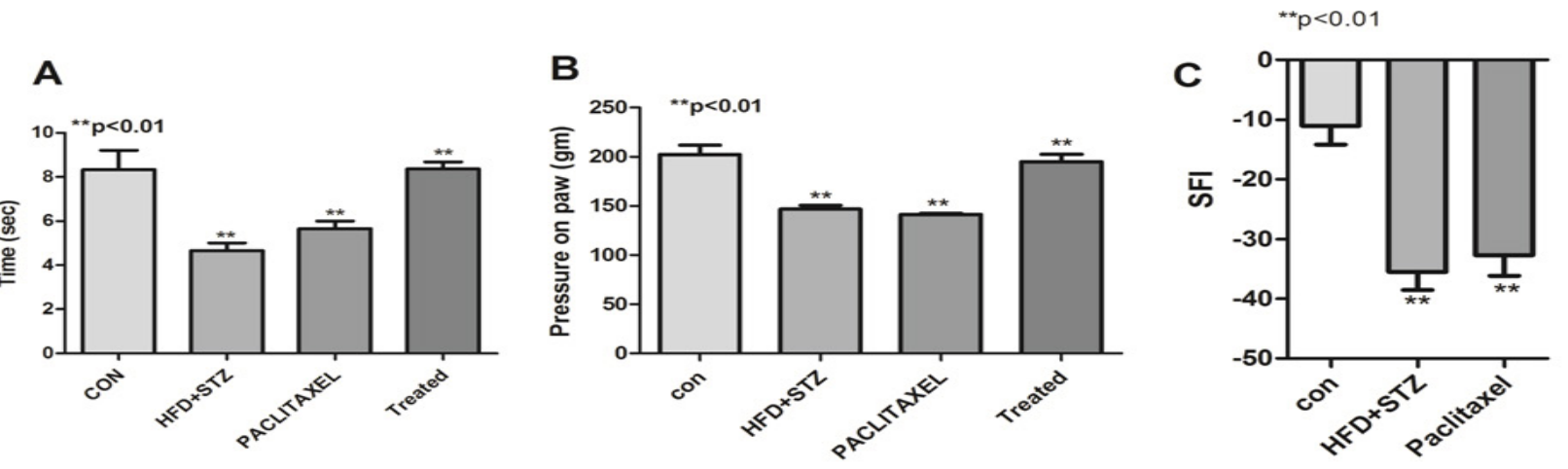

D
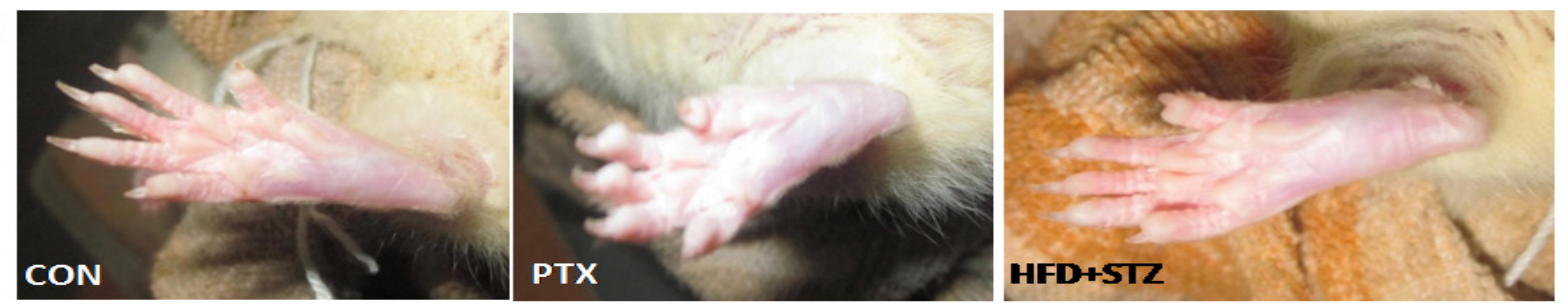

Figure 3: Behavioral neuropathy in type 2 diabetic animals. A) Reaction Time to withdraw/flick tail from hot stimuli. A significant reduction in the reaction time was observed in Paclitaxel $(p<0.05)$ and HFD-STZ $(p<0.05)$ as compared to control animals. No significant change was observed between paclitaxel and HFD-STZ groups. Glibenclamide treatment reversed this decrease in reaction time. B) Paw withdrawal or vocalization threshold using Randall sellito. A significant reduction ( $p<0.01)$ in paw withdrawal threshold was observed in paclitaxel and HFD-STZ animals compared to normal controls. Glibenclamide treatment reversed this decrease in paw withdrawal threshold. C) Sciatic Functional Index (SFI). Significant change $(p<0.01)$ in SFI was observed in paclitaxel and HFD-STZ as compared to control group. D) Morphological changes observed in the hind paw of rats due to the development of neuropathy: In the Control animals, the toe spread and print length were well defined. Also, the distance between $2^{\text {nd }}$ and the $4^{\text {th }}$ toe is not merged. While in the paclitaxel and HFD-STZ animals, the toe spread, and the print length was not as well defined as in control group. All values are expressed as mean $\pm S E M$ for $n=10$. 
A

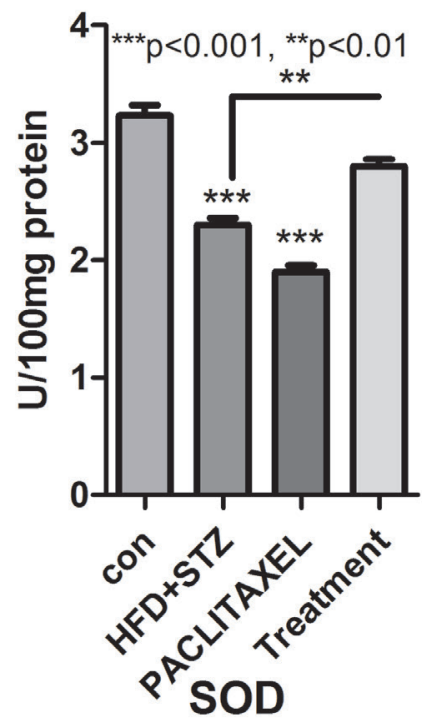

B

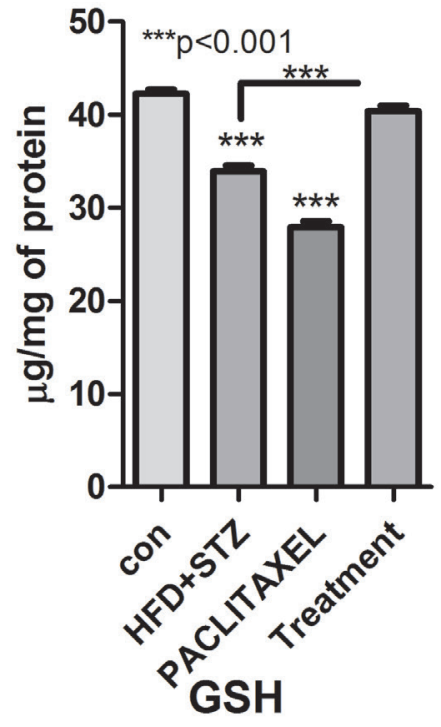

C

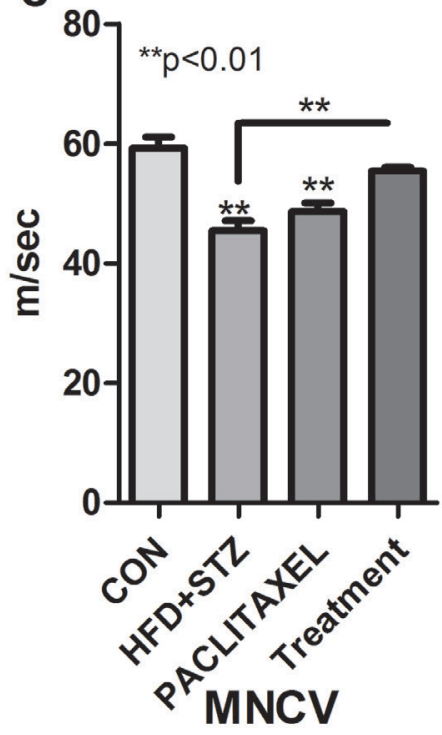

Figure 4: Levels of SOD, GSH, and MNCV in type 2 diabetic animals. A) A significant decrease in SOD levels was found in paclitaxel $(p<0.001)$ and HFD-STZ $(p<0.001)$ compared to control group. Glibenclamide treatment normalized GSH levels. B) A significant reduction in GSH was observed in paclitaxel $(p<0.001)$ and HFD-STZ animals $(p<0.001)$ as compared to control. Glibenclamide treatment normalized GSH levels $C$ ) Motor nerve conduction velocity was significantly decreased ( $p<0.01)$ upon paclitaxel and HFD-STZ treatment as compared to untreated group. All values are expressed as mean \pm SEM for $n=10$.

\section{Histopathological evaluation}

Normal control animals showed the regular arrangement of fibres and there was no axonal swelling observed in this group. The diabetic and paclitaxel group showed derangement of nerve fibres with significant axonal swelling. Sciatic nerves also showed an increase in the number of Schwann cells, an indication of sciatic nerve damage in HFD-STZ and paclitaxel-treated animals as compared to control animals. Glibenclamide treatment could partially reverse the above histological changes upon 3 weeks treatment of diabetic animals. (Figure 5)

\section{DISCUSSION}

In the present study, we attempt to develop a cost efficient murine model of Type 2 diabetic neuropathy which may come close to mimicking the human form of the disease. The initial efforts were directed towards optimizing the rat model for type 2 diabetes. The rats were given a simplified high-fat diet (NPD + Lard) for three weeks followed by a dual dose of STZ $(25 \mathrm{mg} / \mathrm{kg}$ at an interval of 7 days). The animals (90\%) developed elevated blood glucose levels $(>250 \mathrm{mg} / \mathrm{dL}$ fasting) which sustained for ten weeks with little pancreatic $\beta$ cell damage (histopathological data not shown). These animals also developed hypertriglyceridemia and hypercholesterolemia. The preferential use of increased fatty acids for oxidation may blunt the insulin-mediated reduction of hepatic glucose output leading to compensatory hyperinsulinemia, a common feature of insulin resistance..$^{29}$

Both prolonged hyperglycemia and hyperlipidemia are necessary for the development of secondary complications. We proceeded further to evaluate this animal model for the development of neuropathy. To efficiently determine the development of neuropathy in HFDSTZ induced diabetic rats we used paclitaxel-induced neuropathy model as standard. In experimental animals, paclitaxel has been reported to produce dose-dependent painful peripheral neuropathy ${ }^{10}$ Paclitaxel-induced neurotoxicity typically presents as sensory neuropathy with symptoms like numbness, tingling and burning pain. In our study, paclitaxel-induced neuropathy was found to develop in a week and persisted up to 4 weeks.

Major neuropathic behavioral changes measured in HFD-STZ animals constituted thermal hyperalgesia (tail immersion test), ${ }^{30}$ mechanical hyperalgesia (RandallSelitto test), ${ }^{13}$ and gait analysis (gait test). ${ }^{31,32}$ Long-term diabetes is often accompanied with neuropathic pain, and this was evident in our studies by a reduction in tail flick latency and reduced vocalization threshold in Randall-Selitto which was reversed after 3-week glibenclamide treatment. However, if the animals were allowed to be diabetic for 12 weeks, it led to hypoalgesia a sign for late stage neuropathy associated with loss of peripheral nerve fibers. ${ }^{27}$ These hypoalgesic symptoms 

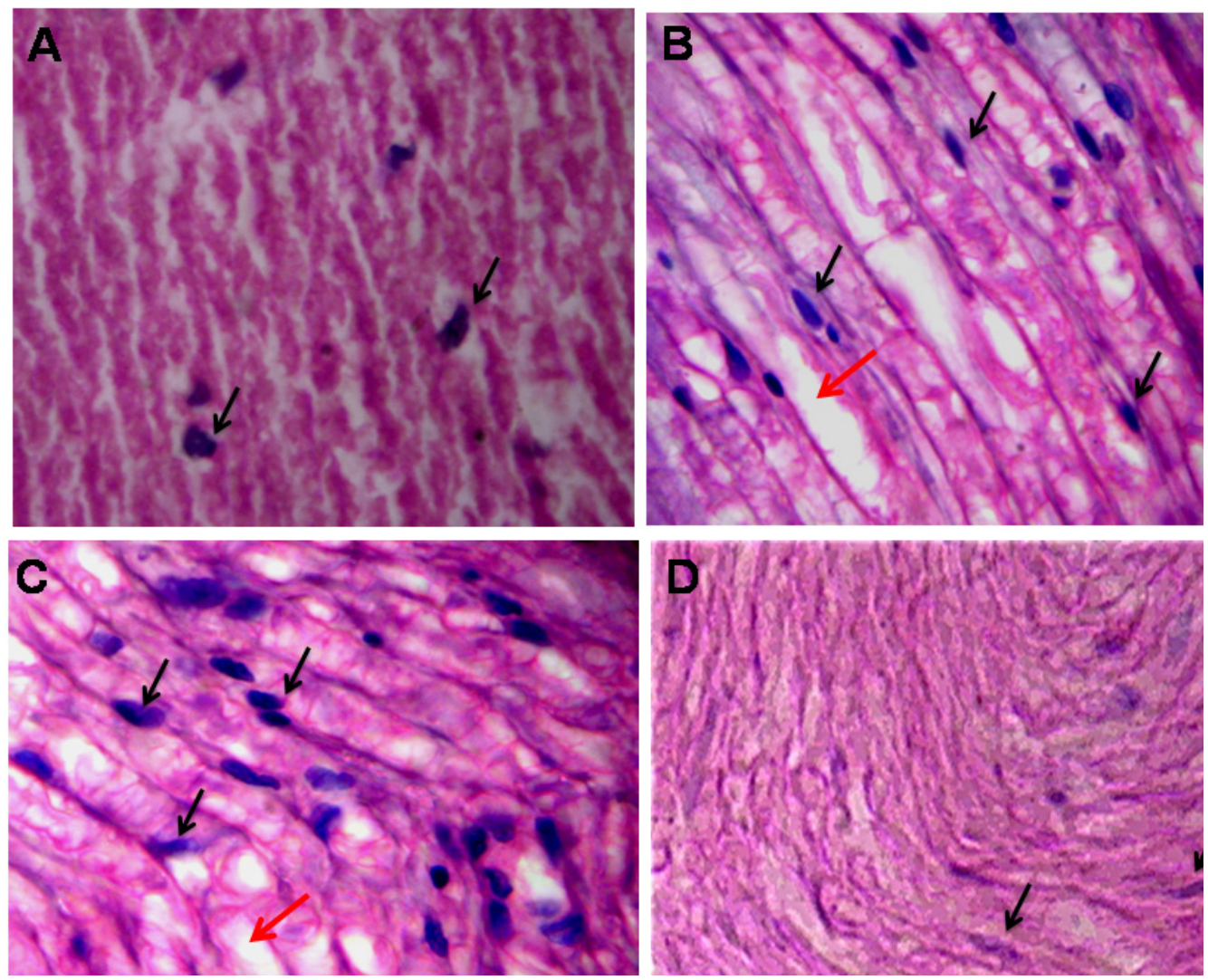

Figure 5: Histopathological evaluation of sciatic nerve of type 2 diabetic animals. A) Control animals showed the normal arrangement of fibers. B) paclitaxel and C) diabetic group showed derangement of nerve fibers with significant axonal swelling and increase in the number of Schwann cells D) Glibenclamide treated diabetic animals showing the normal arrangement of nerve fibers. Representative image of Haematoxylin and Eosin stained sciatic nerves from each treatment group under 10x40X magnification ( $n=6 /$ group).

were not reversed upon glibenclamide treatment. Even the gait test showed highly negative sciatic functional index as well as phenotypic changes in the paws, confirming the extent of nerve damage. These neuropathic behavioral changes in HFD-STZ group were comparable with paclitaxel-treated neuropathy group. Nerve conduction velocity test is a commonly used functional biomarker to assess electrical conduction of motor and sensory nerves of the body. It is known that a healthy nerve conducts impulse with greater speed and strength than a damaged one. Slowing of nerve conductions in limb areas indicate the development of generalized peripheral neuropathy. ${ }^{33}$ We saw a pronounced decrease in nerve conduction velocity in both HFD-STZ and paclitaxel-treated rats compared to non-diabetic animals thus, strongly implying the development of neuropathy. The decline in MNCV was reversed upon glibenclamide treatment. Previous studies show that neuropathy is often accompanied by marked changes in the axonal architecture, number of Schwann cells and arrangement of nerve fibers. The histological studies carried out in the transverse section of the sciatic nerve of diabetic rats, and paclitaxel-treated animals showed axonal swelling, increase in Schwann cells and derangement of nerve fibers while glibenclamide treatment could prevent them in diabetic animals. A considerable body of clinical and experimental evidence suggests the role of freeradical-mediated oxidative processes in the development of diabetic complications. The increase in the production of free radicals can result from the hyperglycemiainduced enhancement in glucose autoxidation, protein glycation, and subsequent oxidative degradation of glycated proteins. ${ }^{34}$ Production of free radicals due to excess oxidation may also be responsible for nerve damage while cellular antioxidants such as superoxide dismutase (SOD), reduced glutathione (GSH) and catalase (CAT) may all play a protective role in this process. ${ }^{35}$ Here we show a considerable decrease in the levels of SOD and GSH in sciatic nerve of diabetic rats when compared to control animals, and glibenclamide treated diabetic animals, further supporting the contention that oxidative stress may be responsible for the progression of neuropathy in these diabetic animals. Mechanisms involved in sulphonylurea mediated protection of peripheral nerves in diabetes are not well characterized. Previous animal studies show sulphonylurea (gliclazide) 
mediated protection from diabetic neuropathy is independent of circulating glucose levels primarily believed to be modulated by preventing DRP-1 mediated oxidative stress as well as by reducing lipid peroxide, and tumor necrosis factor- $\alpha$ production. ${ }^{36-38}$ In our model, we show a glibenclamide mediated increase in SOD and GSH levels which may help in protecting peripheral nerves from oxidative damage. However, future studies need to characterize the associated pathways involved in glibenclamide mediated protection of peripheral nerves.

\section{CONCLUSION}

Hence, a non-transgenic diet and chemical induced animal model of type 2 diabetic neuropathy were developed that closely reflects the characteristics of human type 2 diabetes-associated neuropathy in its early stages and may be helpful in the screening of peripheral neuroprotective molecules especially in resource limited settings.

\section{ACKNOWLEDGEMENT}

We thank Dr R K Sinha and Dr Y Aggarwal (Department of Bioengineering, BIT, Mesra) for extending their support in determining MNCV. The study was funded by DST-SERB project no SB/FT/LS-202/2012.

\section{CONFLICT OF INTERST}

No conflict of interest are declared..

\section{ABBREVIATIONS USED}

HFD: High fat diet; STZ: Streptozotocin; MNCV: Motor Nerve conduction velocity; SOD: Superoxide dismutase; GSH: Glutathione; CAT: Catalase; SD: sprague-dawley.

\section{REFERENCES}

1. Rutkove SB. Electrical impedance myography: background, current state, and future directions. Muscle Nerve. 2009;40(6):936-46. https://doi. org/10.1002/mus.21362 PMid:19768754 PMCid:PMC2824130.

2. Priya S, Kushwaha, Ashok K S, Amit K K, Siddhartha M, Sudipta S. An updated review on the phytochemistry, pharmacology, and clinical trials of Salacia oblonga. 2016;16:109-14.

3. Bhanot A, Shri R. A comparative profile of methanol extracts of Allium cepa and Allium sativum in diabetic neuropathy in mice. Pharmacognosy Res. 2010;2(6):374-84. https://doi.org/10.4103/0974-8490.75460 PMid:21713142 PMCid:PMC3111698.

4. Bugger H, Abel ED. Rodent models of diabetic cardiomyopathy. Dis Model Mech. 2009;2(9-10):454-66. https://doi.org/10.1242/dmm.001941 PMid:19726805.

5. Srinivasan K, Ramarao P. Animal Models in type 2 Diabetes Research: An Overview. Indian J Med Res. 2007;125(3):451-72. PMid:17496368.

6. Reed MJ, Meszaros K, Entes LJ, Claypool MD, Pinkett JG, Gadbois TM, Reaven GM. A new rat model of type 2 diabetes: the fat-fed, streptozotocin- treated rat. Metabolism. 2000;49(11):1390-4. https://doi.org/10.1053/ meta.2000.17721 PMid:11092499.

7. Srinivasan K, Viswanand B, Asrat, Lydia, Kaul CL, Ramarao P. Combination of High Fat Diet Fed and Low dose strptozotocin treated rat: A model for type 2 diabetes and pharmacological screening. Pharmacol Res. 2005;52(4):313-20. https://doi.org/10.1016/j.phrs.2005.05.004 PMid:15979893.

8. Sharma U, Sahu R, Roy A, Golwala D. In vivo Antidiabetic and Antioxidant Potential of Stephania hernandifolia in Streptozotocin-Induced-Diabetic Rats. J Young Pharm. 2010;2(3):255-60. https://doi.org/10.4103/0975-1483.66803 PMid:21042481 PMCid:PMC2964769.

9. Gunnarsson R, Berne C, Hellerstrom C. Cytotoxic effects of streptozotocin and $\mathrm{N}$-nitrosomethylurea on the pancreatic $\mathrm{B}$ cells with special regard to the role of nicotinamide-adenine dinucleotide. Biochem J. 1974;140(3):487-94. https://doi.org/10.1042/bj1400487 PMid:4374939 PMCid:PMC1168026.

10. Zhang M, Lv XY, Li J, Xu ZG, Chen L. The characterization of high-fat diet and multiple low-dose streptozotocin induced type 2 diabetes rat model. Exp diabetes res. 2008;704045. https://doi.org/10.1155/2008/704045

11. Davidson EP, Coppey LJ, Holmes A, Dake B, Yorek MA. Effect of treatment of high fat fed/low dose streptozotocin-diabetic rats with llepatril on vascular and neural complications. Eur J Pharmacol. 2011;668(3):497-506. https:// doi.org/10.1016/j.ejphar.2011.07.016 PMid:21816138 PMCid:PMC3185159.

12. Mansor LS, Gonzalez ER, Cole MA, Tyler DJ, Beeson JH, Clarke K, Carr CA, Heather LC. Cardiac metabolism in a new rat model of type 2 diabetes using high-fat diet with low dose streptozotocin. Cardiovasc Diabetol. 2013;12:136. https://doi.org/10.1186/1475-2840-12-136 PMid:24063408 PMCid:PMC3849358.

13. Scripture CD, Figg WD, Sparreboom A. Peripheral neuropathy induced by paclitaxel: recent insights and future perspectives. Curr Neuropharmacol. 2006; 4(2):165-72. https://doi.org/10.2174/157015906776359568 PMid:18615126 PMCid:PMC2430667.

14. Elvis OA, Eric W, Eric BG, Wonder KMA, James OK, Reimmel KA. Antiallodynic and Anti-hyperalgesic effects of an ethanolic extract and xylopic acid from the fruits of Xylopia aethiopica in murine models of neuropathic pain. 2014;6(2):172-79

15. Bing L, Nicolas A, David B, Alain E, Francois C. Behavioral and Pharmacological description of oxaliplatin-induced painful neuropathy in rat. Pain. 2007;128(3):225-34. https://doi.org/10.1016/j.pain.2006.09.016 PMid:17084975.

16. Santos-Nogoueira E, Redondo Castro E, Mancuso R, Navarro X. Randall Selitto Test: A new approach for the detection of neuropathic pain after spinal cord injury. J Neurotrauma. 2012;29(5):898-904. https://doi.org/10.1089/ neu.2010.1700 PMid:21682605 PMCid:PMC3303094.

17. De Medinaceli L, Freed WJ, Wyatt RJ. An index of the functional conduction of rat sciatic nerve based on measurements made from walking tracks. Exp Neurol. 1982;77(3):634-43. https://doi.org/10.1016/0014-4886(82)90234-5.

18. Gasparini ALP, Barbieri CH, Mazzer N. Correlation between different methods of gait functional evaluation in rats with in sciatic nerve crushing injuries. Acta Ortop Bras. 2007;15(5):285-9.

19. Zhang X, Xin N, Tong L, Tong XJ. Electrical stimulation enhances peripheral nerve regeneration after crush injury in rats. Mol Med Rep. 2013;7(5):1523-7. PMid:23545781.

20. Kumar A, Kaundal RK, lyer B, Sharma SS. Effects of resveratrol on nerve functions, oxidative stress and DNA fragmentation in experimental diabetic neuropathy. Life Sci. 2007;80(13):1236-44. https://doi.org/10.1016/j.lfs.2006. 12.036 PMid:17289084.

21. Moron MS, Depierre JW, Mannervik B. Levels of glutathione, glutathione reductase and glutathione S-transferase activities in rat lung and liver. Biochim Biophys Acta. 1979;582(1):67-78. https://doi.org/10.1016/03044165(79)90289-7.

22. Kaur S, Rana AC, Gangwani S, Sharma R. Punica Granatum attenuates sciatic nerve ligation induced neuropathic pain. Int J Pharm Sci Rev Res. 2012;3(2):509-18.

23. Misra HP, Fridovich I. The role of superoxide anion in the antioxidation of epinephrine and a simple assay for superoxide dismutase. J. Biol. Chem. 1972;247(10):3170-5. PMid:4623845.

24. Arunachalam M, Nirmal S. Attenuating effect of Hydroalcoholic extract of Acorus calamus in Vincristione induced painful neuropathy in rats. J Nat Med. 2011;65:485-7. 
25. Saha L, Hota D, Chakrabarti A. Evaluation of lercanidipine in Paclitaxelinduced neuropathic pain model in rat: a preliminary study. Pain Res Treat. 2012;2012:143579 https://doi.org/10.1155/2012/143579.

26. Mangaiarkkarasi A, Rameshkannan S, Ali RM. Effect of Gabapentin and Pregabalin in Rat Model of Taxol Induced Neuropathic Pain. J Clin Diagn Res. 2015;9(5):FF11-4. https://doi.org/10.7860/jcdr/2015/13373.5955.

27. Shaikh AS, Somani RS. Animal models and biomarkers of neuropathy in diabetic rodents. Indian J Pharmacol. 2010;42(3):129-34. https://doi. org/10.4103/0253-7613.66833 PMid:20871761 PMCid:PMC2937311.

28. Dellon ES, Dellon AL. Functional assessment of neurologic impairment: track analysis in diabetic and compression neuropathies. Plast Reconstr Surg. 1991;88(4):686-94. https://doi.org/10.1097/00006534-199110000-00020.

29. Reaven GM. Insulin resistance, hyperinsulinemia, hypertriglyceridemia and hypertension. Parallels between human disease and rodent models. Diabetes Care. 1991;14(3):195-202. https://doi.org/10.2337/diacare.14.3.195.

30. Kaur G, Singh AJ, Singh N. Exploring the potential effect of Ocimum sanctum in vincristine -induced neuropathic pain in rats. J Brachail Plex Peripher Nerve Inj. 2010;5(1):3. PMid:20181005 PMCid:PMC2832770.

31. Guttmann E, Guttmann L. Factors affecting recovery of sensory function after nerve lesions. J Neurol Neurosurg Psychiatry. 1942;5(3-4):117-29. https:// doi.org/10.1136/jnnp.5.3-4.117 https://doi.org/10.1136/jnnp.5.3-4.81.

32. Sarikcioglu L, Demirel BM, Demir N. Morphological and ultrastructral analysis of the water shed zones after stripping of the vasa nervorum, Int $\mathrm{J}$ Neurosci. 2008;118(8):1145-55. https://doi.org/10.1080/00207450801898220 PMid:18576212.

\section{Pictorial Abstract}

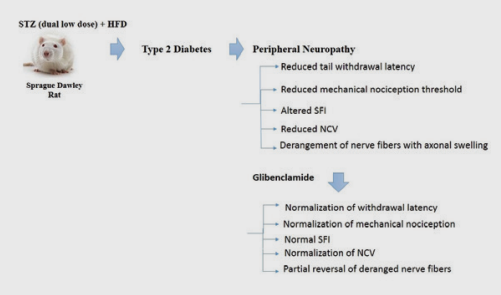

33. Tomlinson DR, Moriarty RJ, Mayer JH. Prevention and reversal of defective axonal transport and motor nerve conduction velocity in rats with experimental diabetes by treatment with the aldose reductase inhibitor Sorbinil. Diabetes. 1984;33(5):470-6. https://doi.org/10.2337/diabetes.33.5.470 https://doi. org/10.2337/diab.33.5.470 PMid:6202576.

34. Wolff SP, Dean RT. Glucose autoxidation and protein modification. The potential role of 'autoxidative glycosylation' in diabetes. Biochem J. 1987;245(1):243-50. https://doi.org/10.1042/bj2450243.

35. Kasznicki J, Kosmalski M, Sliwinska A, Mrowicka M, Stancyzk M, Majsterek I, Drzewoski J. Evaluation of oxidative stress markers in the pathogenesis of diabetic neuropathy. Mol Biol Rep. 2012;39(9):8669-78. https://doi. org/10.1007/s11033-012-1722-9 PMid:22718504 PMCid:PMC3404273.

36. Qiang X, Satoh J, Sagara M, Fukuzawa M, Masuda T, Miyaguchi S, Takahashi K, Toyota T. Gliclazide inhibits diabetic neuropathy irrespective of blood glucose levels in streptozotocin-induced diabetic rats. Metabolism. 1998;47(8):977-81. https://doi.org/10.1016/S0026-0495(98)90354-7.

37. Wu YB, Shi LL, Wu YJ, Xu WH, Wang L, Ren MS. Protective effect of gliclazide on diabetic peripheral neuropathy through Drp-1 mediatedoxidative stress and apoptosis. Neurosci Lett. 2012;523(1):45-9 https://doi. org/10.1016/j.neulet.2012.06.038 PMid:22732450.

38. Attia HN, Al-Rasheed NM, Al-Rasheed NM, Maklad YA, Ahmed AA, Kenawy SA. Protective effects of combined therapy of gliclazide with curcumin in experimental diabetic neuropathy in rats. Behav Pharmacol. 2012;23(2):153-61 https://doi.org/10.1097/FBP.0b013e3283512c00 PMid:22411174.

\section{SUMMARY}

A non-transgenic diet and chemical induced animal model of type 2 diabetic neuropathy were developed and characterised. The model closely reflects the characteristics of human type 2 diabetes-associated neuropathy in its early stages and may be helpful in the screening of peripheral neuroprotective molecules especially in resource limited settings.

\section{About Authors}

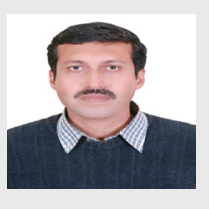

Dr. Sugato Banerjee: Is working as Assistant Professor in the Department of Pharmaceutical Sciences and Technology, BIT Mesra, Ranchi, India. He has published more than 30 peer-reviewed research articles in international journals. He is an editorial board member of Pharmacognosy Magazine. His primary research area is to study secondary complications and their underlying mechanisms associated with Type 2 diabetes using non-transgenic murine models.

Cite this article: Mehta BK, Nerkar D, Banerjee S. Characterization of Peripheral Neuropathy in Rat Model of Type 2 Diabetes. Indian J of Pharmaceutical Education and Research. 2017;51(1):92-101. 